\title{
Carbono acumulado en raíces de especies vegetales en sistemas silvopastoriles en el Norte de Colombia
}

\author{
Carbon accumulated in roots of plant species in silvopastoral systems in northern \\ Colombia
}

\author{
Jose Luis Contreras-Santos ${ }^{1}$, Judith Martínez-Atencia ${ }^{2}$, Cindy Katherine Falla-Guzman ${ }^{3}$
}

[Recibido: 23 de noviembre 2019, Aceptado: 21 de mayo 2020, Corregido: 26 de agosto 2020, Publicado: 1 de enero 2021]

\section{Resumen}

[Introducción]: Los sistemas silvopastoriles (SSP) son alternativas sostenibles en el manejo de suelos dedicados a la actividad ganadera, que contribuyen a la recuperación de suelos degradados. [Objetivo]: Conocer el potencial de captura de carbono (C) en biomasa de raíces finas por especie y su capacidad de exploración en el suelo dentro de dos SSP (A1 - Megathyrsus maximum cv Mombasa asociada con las especies arbustivas Leucaena leucocephala y Crescentia cujete; A2 - combinación de A1 + especies arbóreas Guazuma ulmifolia, Cassia grandis y Albizia saman) y pradera sin árboles (A0 - M. maximum cv Mombasa), en el valle medio del río Sinú. [Metodología]: Se empleó un diseño de bloques completos al azar con 3 tratamientos y 3 repeticiones, sobre los cuales se determinó biomasa de raíces finas $(\mathrm{BRF}, \leq 5 \mathrm{~mm})$ con cilindro de volumen conocido y densidad de longitud de raíz (DLR) mediante escáner de superficie plana, igualmente se determinaron propiedades fisicoquímicas de suelo. [Resultados]: La L. leucocephala mostró valores altos de C acumulado en la biomasa de raíces (441.5 kg C ha 1) respecto a las otras especies evaluadas. En contraste, el $\mathrm{C}$ acumulado en raíces de la gramínea se vio afectado por la complejidad de los SSP, reduciéndose el $59 \%$ (A1) y 34 \% (A2), respecto A0. La mayor DLR se registró en las especies C. cujete y L. leucocephala $\left(249.7 \pm 199.5\right.$ y $239.3 \pm 135.02 \mathrm{~cm} \mathrm{~cm}^{-2}$, respectivamente). [Conclusiones]: Los SSP acumulan mayor C en biomasa de raíces y mejoran las propiedades fisicoquímicas del suelo.

Palabras clave: Densidad de longitud; gramínea; propiedades fisicoquímicas; raíces finas; suelo.

\section{Abstract}

[Introduction]: Silvopastoral systems (SPSs) are sustainable alternatives to manage soils dedicated to livestock production, contributing to the recovery of degraded soils. [Objective]: Know the potential of carbon capture (C-capture) in fine root biomass per species and their exploration capacity in the soil between two SPSs (A1 - Megathyrsus maximum cv Mombasa associated with the shrub species, Leucaena leucocephala and Crescentia cujete; A2 - combination of A1 + tree species, Guazuma ulmifolia, Cassia grandis, and Albizia saman) and meadows without trees (A0 - M. maximum cv Mombasa), in the middle valley of the Sinú River. [Methodology]: A randomized complete block design was used with three treatments and three repetitions, in which fine root biomass (FRB, $\leq$

1 Investigador, Corporación Colombiana de Investigación Agropecuaria (AGROSAVIA), Colombia, jlcontreras@agrosavia.co; https://orcid.org/0000-0002-8179-3430

2 Investigadora, Corporación Colombiana de Investigación Agropecuaria (AGROSAVIA), Colombia, jcmartinez@agrosavia.co; https://orcid.org/0000-0002-1266-8505

3 Asistente de investigación, Corporación Colombiana de Investigación Agropecuaria (AGROSAVIA), Colombia, cfalla@agrosavia.co; https://orcid.org/0000-0002-8179-3430

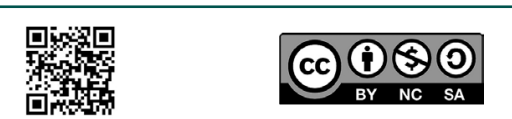

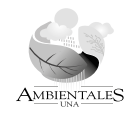




\section{Revista de CIENCIAS AMBIENTALES Tropical Journal of Environmental Sciences}

Revista de Ciencias Ambientales (Trop J Environ Sci) e-ISSN: 2215-3896 (Enero-Junio, 2021). Vol 55(1): 52-69 DOI: https://doi.org/10.15359/rca.55-1.3

Open Access: www.revistas.una.ac.cr/ambientales e-mail: revista.ambientales@una.ac.cr Contreras-Santos J . L., Martínez-Atencia J., Falla-Guzman C. K.

$5 \mathrm{~mm}$ ) was determined with a cylinder of known volume and root length density (RLD) using a flatbed scanner; physicochemical properties of soil were also determined. [Results]: L. leucocephala showed high values of $\mathrm{C}$ accumulation in root biomass ( $441.5 \mathrm{~kg} \mathrm{Cha}^{-1}$ ) compared to the other species evaluated. In contrast, $\mathrm{C}$ accumulated in grass roots was affected by the SPS complexity, reducing $59 \%$ (A1) and $34 \%$ (A2), with respect to A0. The highest RLD was recorded in the C. cujete and L. leucocephala species $\left(249.7 \pm 199.5\right.$ and $239.3 \pm 135.02 \mathrm{~cm} \mathrm{~cm}^{-2}$, respectively). [Conclusions]: SPSs accumulate higher $C$ in root biomass and improve the soil's physicochemical properties.

Keywords: Fine roots; grass; length density; physicochemical properties; soil.

\section{Introducción}

En las últimas décadas, la demanda por alimento ha aumentado, a causa del crecimiento demográfico y económico, lo cual ha conllevado a la conversión de áreas boscosas en sistemas de producción agropecuarios, asociados a malas prácticas de manejo que han sido los causantes de más del $68 \%$ de la degradación de los suelos en América Latina y el Caribe (FAO y GTIS, 2015).

El suelo es el principal reservorio de carbono (C) en los ecosistemas terrestres; el cambio en su uso, asociado a procesos de degradación, es la principal causa de la reducción de $\mathrm{C}$ dentro de este. Acarrea aumento en las emisiones de dióxido de carbono $\left(\mathrm{CO}_{2}\right)$ hacia la atmosfera, y cambia su funcionalidad como sumidero (Céspedes et al., 2012; Pérez-Ramírez et al., 2013).

Sin embargo, en los últimos años la tendencia mundial ha sido buscar alternativas que ayuden a reducir los procesos de degradación del suelo, agua y aire, generando servicios ecosistémicos y adaptabilidad de los sistemas productivos agrícolas y pecuarios a los cambios climáticos actuales. Como alternativa sostenible para contrarrestar estos efectos, los sistemas agroforestales (SAF) y silvopastoriles (SSP) son propuestas económicas y ambientales viables a corto y largo plazo (Contreras-Santos et al., 2019; Sotelo et al., 2017). Diferentes estudios han resaltado las bondades de asociar especies gramíneas con especies arbustivas, arbóreas, frutales y maderables (sistemas silvopastoriles o agroforestales) capaces de aportar grandes volúmenes de materia orgánica (M.O) a través de la acumulación de hojarasca y raíces finas, para generar condiciones fisicoquímicas y biológicas del suelo adecuadas para el desarrollo de los cultivos (Sotelo et al., 2017).

Conocer los servicios ecosistémicos que aportan los sistemas silvopastoriles (SSP) o agroforestales (SAF) conlleva a un mejor entendimiento de la dinámica de las especies vegetales que son parte de estos. Autores como Rojas et al. (2009) reportan la importancia de evaluar la producción y distribución de las raíces dentro de estos sistemas, con el fin de conocer su potencial en la acumulación de carbono orgánico. Igualmente, José y Dollinger (2019) en la revisión de 28 documentos encontraron la importancia de conocer los beneficios ecosistémicos de los componentes de los sistemas silvopastoriles, asociados a la captura de carbono.

El estudio de las raíces finas $(\leq 5 \mathrm{~mm})$ dentro de sistemas simultáneos es de gran importancia, debido a que en ellos ocurren interacciones entre las diversas especies. Las raíces finas son las estructuras principalmente responsables de la absorción de agua y nutrientes en las plantas (Baker et al., 2002; Bowen, 1985); por esta razón, conocer las ventajas y desventajas de los

\begin{tabular}{|c|c|c|}
\hline 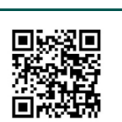 & (c) (1) (\$) () () & 53 \\
\hline
\end{tabular}




\section{Revista de CIENCIAS AMBIENTALES Tropical Journal of Environmental Sciences}

Revista de Ciencias Ambientales (Trop J Environ Sci) e-ISSN: 2215-3896 (Enero-Junio, 2021). Vol 55(1): 52-69 DOI: https://doi.org/10.15359/rca.55-1.3

Open Access: www.revistas.una.ac.cr/ambientales e-mail: revista.ambientales@una.ac.cr Contreras-Santos J . L., Martínez-Atencia J., Falla-Guzman C. K.

sistemas radiculares de las distintas especies y su cambio en el espacio es necesario para la optimización de los asocios agroforestales o silvopastoriles (Schroth y Zech, 1995).

Distintos estudios han demostrado la importancia de las raíces finas (RF) para el funcionamiento del ecosistema, no solo por su participación en la productividad primaria neta (PPN), sino también en los ciclos biogeoquímicos de los bosques, debido a que su descomposición contribuye significativamente al enriquecimiento de los horizontes orgánicos por su rápida reconversión (Burke y Raynal, 1994).

La BRF permite conocer la distribución de las raíces sobre el perfil del suelo, al igual que la fijación de carbono en el ecosistema, producto del recambio de raíces (Casals et al., 2014), así mismo es un indicador de la capacidad de cada especie de planta en la obtención de nutrimentos y agua (Baker et al., 2002). DLR, expresada en unidad de área específica, permite conocer la relación entre el espacio poroso, movimiento del agua y nutrientes en el suelo (Barreto - Sánchez et al., 2005; Hertel et al., 2003). Estos parámetros son importantes al momento de analizar la dinámica de los nutrientes y el carbono en el suelo (Silver y Miya, 2001).

El objetivo general de este estudio fue determinar el aporte por especie de raíces finas dentro de dos sistemas silvopastoriles (SSP) contrastados con una pradera sin árboles (Pm), de trece años, ubicados en el valle medio del río Sinú.

\section{Metodología}

\subsection{Localización}

El estudio se realizó en el área de Sistemas Silvopastoriles (SSP) de estratos múltiples, ubicado en el Centro de Investigación Turipaná de AGROSAVIA ( $8^{\circ} 51^{\prime}$ de latitud Norte, $75^{\circ} 47^{\prime}$ de longitud Oeste), municipio Cereté, departamento Córdoba, con una altitud de $15 \mathrm{~m}$ s.n.m. Las precipitaciones en la zona de estudio presentan comportamiento unimodal biestacional, un periodo de lluvias de mayo a noviembre y seco de diciembre a marzo, con temperatura promedio anual de $28{ }^{\circ} \mathrm{C}$, humedad relativa de $82 \%$ y precipitación promedio anual de $1200 \mathrm{~mm}$. Según la clasificación de Holdridge (2000), el área de estudio pertenece a la zona agroecológica Bosque Seco Tropical (BsT).

El suelo del área experimental corresponde a un Vertic Endoaquepts, perteneciente a la serie La Pozona, el cual presenta predomino de textura arcillosas, de tipo expansiva (tipo 2:1), régimen de humedad údico y temperatura isohipertérmico, con secuencia de horizontes $\mathrm{Ap}, \mathrm{Bg}$, Bg2 y Cg (Figura 1).

\begin{tabular}{|c|c|c|}
\hline 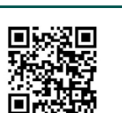 & (c) (1) () () () & 54 \\
\hline
\end{tabular}




\section{Revista de CIENCIAS AMBIENTALES Tropical Journal of Environmental Sciences}

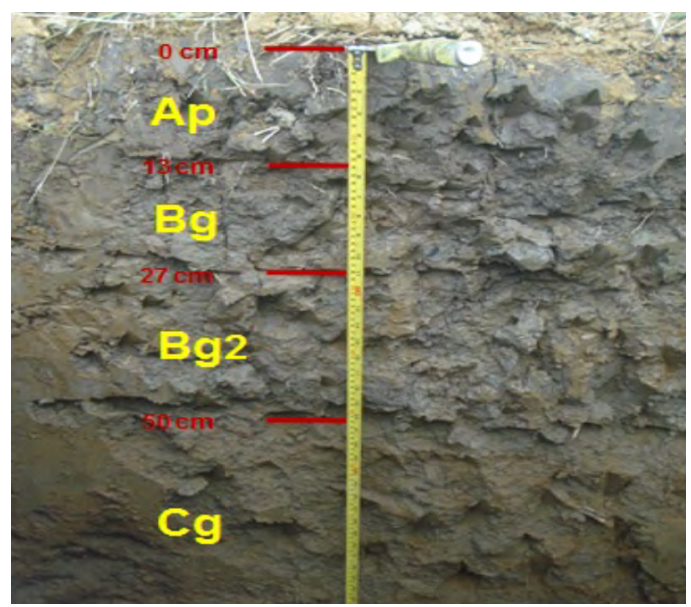

Figura 1. Perfil modal del área de evaluación.

Figure 1. Modal profile of the evaluation area.

\subsection{Tratamientos}

Los arreglos silvopastoriles y pradera sin árboles (Cuadro 1) fueron establecidos en el año de 1998, diseñados con base en la investigación participativa de productores de la región Caribe Colombiana, registrando el conocimiento local de las prácticas silvopastoriles y árboles dispersos utilizadas por los ganaderos de la región (Cajas y Sinclair, 2002). A partir de esto se definieron tres modelos silvopastoriles (SP) asociados con una gramínea adaptada. Igualmente se precisó la funcionalidad de cada especie incluida dentro de los sistemas SP. Para el caso de Leucaena leucocephala y Crescentia cujete se determinó su funcionalidad como banco de proteína de ramoneo directo (arbustivas forrajeras) para la alimentación del ganado vacuno, en épocas críticas, y su altura no supero 1.5 metros.

Cuadro 1. Descripción de tratamientos establecidos en Centro de Investigaciones Turipaná - AGROSAVIA, Cereté-Córdoba.

Table 1. Description of treatments established at the Turipaná Research Center - AGROSAVIA, Cerete - Córdoba, 2012

\begin{tabular}{ll}
\hline Arreglos (A) & Especies \\
\hline Arreglo 0 (A0) & Megathyrsus maximum cv Mombasa (Pm) \\
Arreglo 1 (A1) & Pm asociado con arbustos forrajeros Leucaena leucocephala (Ll) y \\
& Crescentia cujete $(\mathrm{Cc})$. \\
Arreglo 2 (A2) & Pm asociado con arbustos forrajeros Ll, Cc y árboles forrajeros \\
& Guazuma ulmifolia $(\mathrm{Gu})$, Cassia grandis $(\mathrm{Cg})$ y Albizia saman (As) \\
\hline
\end{tabular}

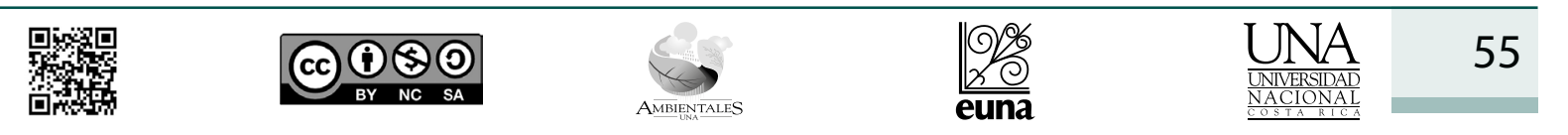




\section{Revista de CIENCIAS AMBIENTALES Tropical Journal of Environmental Sciences}

Revista de Ciencias Ambientales (Trop J Environ Sci) e-ISSN: 2215-3896 (Enero-Junio, 2021). Vol 55(1): 52-69 DOI: https://doi.org/10.15359/rca.55-1.3

Open Access: www.revistas.una.ac.cr/ambientales e-mail: revista.ambientales@una.ac.cr Contreras-Santos J . L., Martínez-Atencia J., Falla-Guzman C. K.

Las especies se establecieron en arreglos de 16 metros $(\mathrm{m})$ entre plantas por 16 metros (m) entre líneas, intercalados sobre y entre líneas de siembra, hasta completar el área de la unidad experimental (100 x 200 m). El total del área de investigación fueron 18 ha.

\subsection{Variables de la raíz fina $(<5 \mathrm{~mm})$}

\subsubsection{Biomasa de raíces (BRF) y densidad de longitud de raíces finas (DLR)}

Durante los meses de mayo a junio de 2012 (periodo lluvioso), se colectaron, aleatoriamente, muestras de raíces finas (RF) de cada especie por triplicado, presente en los arreglos silvopastoriles y pradera sin árboles. Las RF de las especies se obtuvieron por extracción mediante anillo metálico de $10.1 \mathrm{~cm}$ de alto por $9.7 \mathrm{~cm}$ de diámetro interno, para un volumen de $746.37 \mathrm{~cm}^{3} \mathrm{de}$ suelo muestreado (Jiménez y Arias, 2004). Las muestras se conservaron en refrigeración a 4-5 ${ }^{\circ} \mathrm{C}$ hasta su procesamiento, con el fin de evitar pérdidas por descomposición de las RF (Rojas et al., 2009). Posteriormente, las muestras fueron lavadas con agua a presión, con el fin de obtener las raíces con diámetro menor de 5 milímetros $(\mathrm{mm})$, luego de obtenidas las RF, se realizó una clasificación de acuerdo con su estado con proceso de necrosis y sin este, las RF muertas (con procesos de necrosis) no fueron tomadas en cuenta en este estudio.

Luego de la extracción en laboratorio de las RF, las muestras se rotularon y procesaron por medio del software WinRHIZO PRO 3.9, con el fin de obtener el parámetro morfológico de raíz DLR. Posteriormente, estas muestras se secaron en estufa a $70^{\circ} \mathrm{C}$ durante un periodo de 48 horas, para determinar su peso seco (ISO 6496, 2016). Con el fin de contabilizar las pérdidas de materia seca por especie se utilizó un factor de conversión de 1.5 (Rojas et al., 2009). El cálculo de carbono acumulado (CB) en la biomasa de raíces finas se estimó con la BRF multiplicado por el factor de conversión de 0.45 , para lo cual se estimó que el carbono en la biomasa equivale entre el 45 al 50 \% (Céspedes et al., 2012; Quiceno-Urbina et al., 2016).

\subsubsection{Toma de muestra en campo}

Se tomaron muestras de suelo-raíz por triplicado en cada especie a las profundidades 0 a 10 $\mathrm{cm}, 10$ a $20 \mathrm{~cm}$ y 20 a $30 \mathrm{~cm}$, en puntos de observación ubicados entre 30 y $40 \mathrm{~cm}$ del eje central de cada planta muestreada de Ll, Cc y Pm, y entre 70 y $80 \mathrm{~cm}$ para el caso de las especies $\mathrm{Gu}$, As y Cg.

\subsection{Variables fisicoquímicas del suelo}

Se tomaron cinco puntos de observación en la zona central de cada unidad experimental, donde se recogieron muestras de suelo a las profundidades $0-10 \mathrm{~cm}$ y $10-20 \mathrm{~cm}$. La densidad aparente del suelo $(\mathrm{Da})$ se determinó por el método del cilindro de volumen conocido (soil core), densidad real (Dr) por el método del picnómetro, y porosidad total del suelo por la

\begin{tabular}{|c|c|c|}
\hline 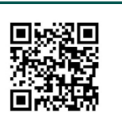 & (c) (1) (1) (2) & 56 \\
\hline
\end{tabular}




\section{Revista de CIENCIAS AMBIENTALES Tropical Journal of Environmental Sciences}

Revista de Ciencias Ambientales (Trop J Environ Sci) e-ISSN: 2215-3896 (Enero-Junio, 2021). Vol 55(1): 52-69 DOI: https://doi.org/10.15359/rca.55-1.3

Open Access: www.revistas.una.ac.cr/ambientales e-mail: revista.ambientales@una.ac.cr Contreras-Santos J . L., Martínez-Atencia J., Falla-Guzman C. K.

relación entre Da y Dr (IGAC, 2006). Los parámetros químicos de suelo se tomaron alrededor de cada punto por quintuplicado, a $20 \mathrm{~cm}$ de profundidad. Posteriormente, estas muestras se homogenizaron por punto para su análisis en el laboratorio de suelos y agua de AGROSAVIA y determinar $\mathrm{pH}$ (potenciométrico, relación 1:1 $\mathrm{P} / \mathrm{V}$ ); $\mathrm{MO}(\%)$ por el método de oxidación de Walkley-Black; fósforo - P (Bray II modificado); Azufre - S extracción con fosfato de calcio monobásico; bases intercambiables $(\mathrm{K}, \mathrm{Ca}, \mathrm{Mg}, \mathrm{Na})$ por acetato de amonio $1 \mathrm{M}$ a $\mathrm{pH}$ 7; capacidad de intercambio catiónico (CIC).

\subsection{Análisis estadístico}

Se usó un diseño de bloques completos al azar, con 3 arreglos y tres repeticiones. El factor de bloqueo correspondió al drenaje (rápido, moderado y lento); para el análisis de las variables morfológicas de raíces (BRF y DLR) y variables fisicoquímicas (Da, Pt y fertilidad completa) de suelo se analizaron mediante una ANOVA de efectos principales y efectos combinados utilizando PROC GLM; las correlaciones de las variables fisicoquímicas de suelo y morfológicas de las raíces (BRF y DLR) se realizaron mediante correlación lineal simple de Pearson del programa estadístico SAS Systems versión 9.2, en los casos en los cuales se detectaron diferencias significativas a un nivel de $5 \%$, se realizaron pruebas de separación de medias, utilizando la prueba HSD de Tukey.

\section{Resultados}

\subsection{Carbono acumulado en biomasa de raíces finas (CBRF)}

El carbono acumulado en la biomasa de raíces finas (BRF) de las especies dentro de los arreglos silvopastoriles (SSP) y pradera sin árboles evidenció diferencias estadísticas significativas $(\mathrm{p}<0.02)$. La gramínea $(\mathrm{PM})$ presentó disminución en el contenido de CBRF a medida que aumentó la complejidad del arreglo SSP, con reducción del 59 \% (A1) y 34 \% (A2), respecto al A0. Similar comportamiento evidencia L. leucocephala $(\mathrm{Ll})$ con reducción en la CBRF del 45 $\%$, cuando incrementó el número de especies dentro del A2. La especie C. cujete (Cc) mantuvo la CBRF dentro de los arreglos evaluados, lo cual indica la capacidad del Cc en competir por nutrientes y agua en el suelo (Figura 2).

\begin{tabular}{|c|c|c|}
\hline 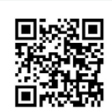 & (c) (1) (9) & 57 \\
\hline
\end{tabular}




\section{Revista de CIENCIAS AMBIENTALES Tropical Journal of Environmental Sciences}

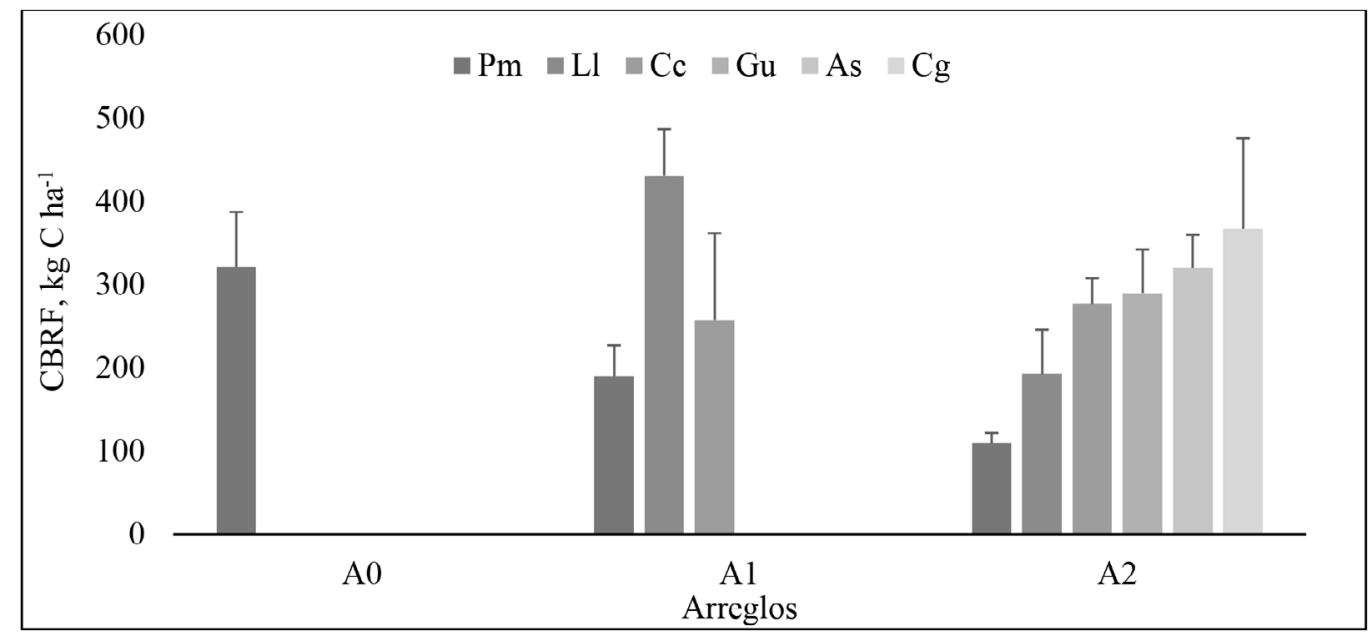

Figura 2. Carbono acumulado en biomasa de raíces finas (CBRF) de las diferentes especies dentro los arreglos silvopastoriles y pradera sin árboles. A0: Pm (gramínea M. maximus cv Mombasa); A1: Pm asociado con arbustos forrajeros $\mathrm{Ll}$ (L. leucocephala), Cc (C. cujete), A2: Pm asociado con arbustos forrajeros Ll, Cc y árboles forrajeros $\mathrm{Gu}$ (G. ulmifolia), $\mathrm{Cg}$ (C. grandis), As (A. saman). Las barras grises representan las medias de las especies dentro de cada arreglo, letras iguales no significancia, línea negra error experimental (EE).

Figure 2. Carbon accumulated in roots biomass (CBFR) of the different species evaluated within silvopastoral arrangements and treeless pasture. A0: Pm (Grass M. maximus cv Mombasa), A1: Pm associated with shrub species Ll ( L. leucocephala), Cc (C. cujete), A2: Pm associated with shrub species Ll and Cc plus tree species Gu (G. ulmifolia), $\mathrm{Cg}$ (C. grandis), As (A. saman). Gray bars represent the means of the species within each arrangement, equal letters not significant, black line experimental error (EE).

Los arreglos que asocian la gramínea con especies arbustivas (A1) y gramínea con especies arbóreas (A2) presentaron los mayores valores de CBRF (7 917.9 \pm 231.1 y $14035 \pm 186.24 \mathrm{~kg} \mathrm{C}$ ha $^{-1}$, respectivamente) frente al A0 $\left(2893.7 \pm 199.25 \mathrm{~kg} \mathrm{C} \mathrm{ha}^{-1}\right)$ hasta los de $30 \mathrm{~cm}$. La interacción arreglo por profundidad fue significativa $(\mathrm{p}<0.05)$, mostró la mayor acumulación de CBRF en la primera capa de suelo $(0-10 \mathrm{~cm})$ en todos los arreglos. En A0 y A1 el CBRF presentó tendencia a disminuir con la profundidad en $53 \%(10-20 \mathrm{~cm})$ y $73 \%(20-30 \mathrm{~cm})$, respecto a lo reportado en los primeros $10 \mathrm{~cm}$ (Figura 3). El arreglo A2 mostró igual CBRF entre 0-10 y 10-20 cm de profundidad. Posteriormente, la tendencia fue a disminuir $(\sim 40 \%)$ con relación con los valores obtenidos en los primeros $20 \mathrm{~cm}$ (Figura 3). 


\section{Revista de CIENCIAS AMBIENTALES Tropical Journal of Environmental Sciences}

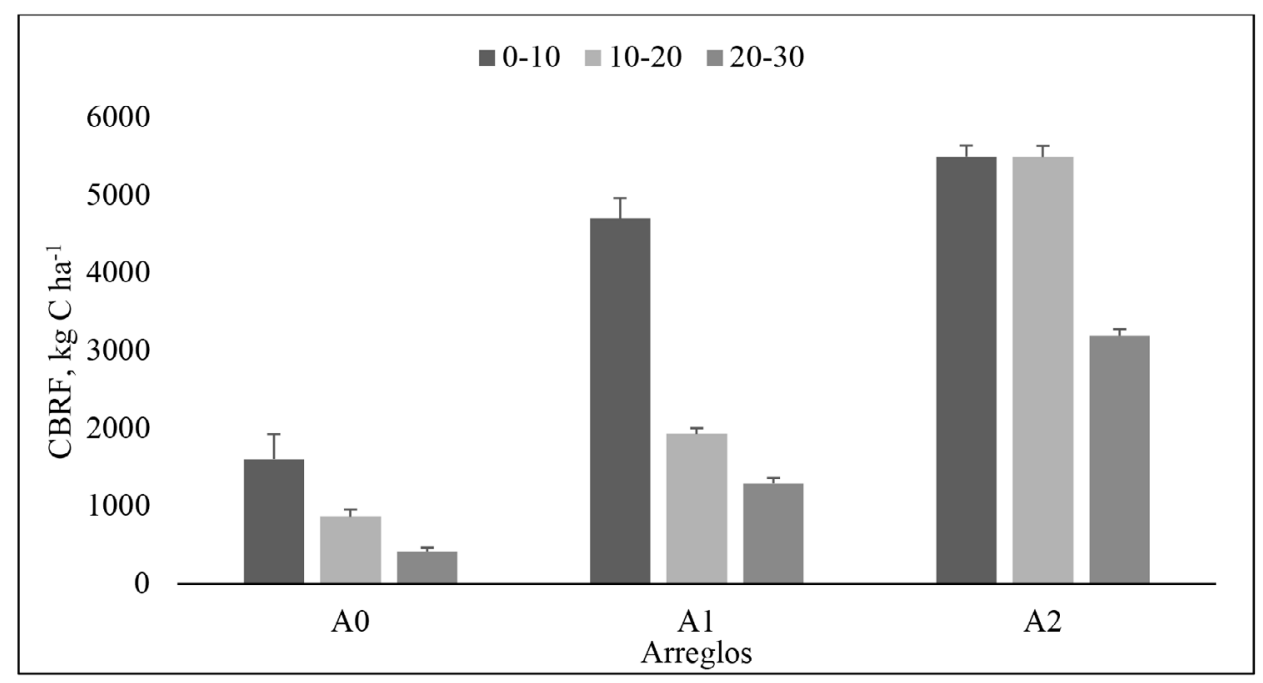

Figura 3. Comportamiento del carbono acumulado en la biomasa de raíces finas (CBRF) en cada arreglo y una paradera sin árboles vs profundidad de suelo, A0: Pm (gramínea M. maximus cv Mombasa); A1: Pm asociado con arbustos forrajeros Ll (L. leucocephala), Cc (C. cujete), A2: Pm asociado con arbustos forrajeros Ll, Cc y árboles forrajeros $\mathrm{Gu}$ (G. ulmifolia), Cg (C. grandis), As (A. saman). Las barras grises representan las medias de las especies evaluadas dentro de cada arreglo, letras iguales no significancia, línea negra error experimental (EE)

Figure 3. CBFR behavior for each arrangement and treeless pasture vs. ground depth. A0: Pm (Grass M. maximus cv Mombasa), A1: Pm associated with shrub species Ll (L. leucocephala), Cc (C. cujete), A2: Pm associated with shrub species Ll and Cc plus tree species Gu (G. ulmifolia), Cg (C. grandis), As (A. saman). Gray bars represent the means of the species evaluated within each arrangement, equal letters not significant, black line experimental error (EE)

\subsection{Densidad de longitud de raíces finas (DLR)}

Los resultados del análisis de varianza evidenciaron diferencias estadísticas altamente significativas $(\mathrm{p}<0.0001)$ en la DLR entre las especies dentro de cada arreglo y la profundidad. El comportamiento de la DLR en la gramínea $(\mathrm{Pm})$ mostró comportamiento similar al reportado en CBRF, es decir, disminuye a medida que aumenta la complejidad de los arreglos. Para el caso del arreglo A1, las especies arbustivas (C. cujete - Cc y L. leucocephala - Ll), presentaron los valores más elevados de DLR, con $249.7 \pm 199.5$ y $239.3 \pm 135.02 \mathrm{~cm} \mathrm{~cm}^{-2}$, respectivamente. El comportamiento de las especies vegetales dentro del arreglo A2 fue similar entre ellas (132.5 \pm 70.42 a $148 \pm 45.84 \mathrm{~cm} \mathrm{~cm}^{-2}$ ) (Figura 4).

\begin{tabular}{|c|c|c|c|c|c|}
\hline 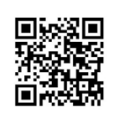 & (c) (1) (9) & $\underset{\text { AMBENTALES }}{\leftrightarrow}$ & $\frac{9 \%}{2 \%}$ & $\frac{\text { UNA }}{\frac{\text { UNIIERSIDAD }}{\text { NASIONAL }}}$ & 59 \\
\hline
\end{tabular}




\section{Revista de CIENCIAS AMBIENTALES

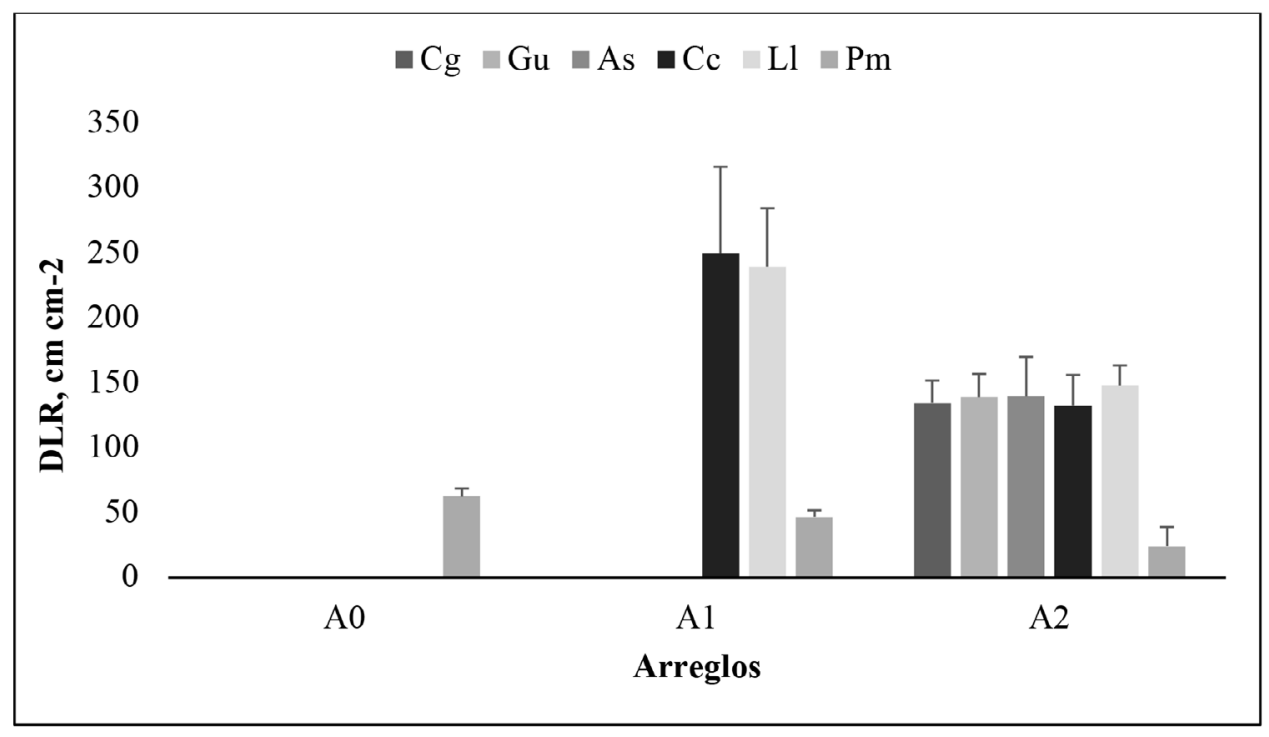

Figura 4. Densidad de longitud de raíces finas (DLR) de las diferentes especies dentro los arreglo silvopastoriles y pradera sin árboles. A0: Pm (Gramínea M. maximus cv Mombasa); A1: Pm asociado con arbustos forrajeros Ll (L. leucocephala), Cc (C. cujete), A2: Pm asociado con arbustos forrajeros Ll, Cc y árboles forrajeros Gu (G. ulmifolia), $\mathrm{Cg}$ (C. grandis), As (A.saman). Las barras grises representan las medias de las especies evaluadas dentro de cada arreglo, letras iguales no significancia, línea negra error experimental (EE)

Figure 4. Fine root length density (RLD) of the different species within the silvopastoral and grassland arrangements without trees. A0: Pm (Grass M. maximus cv Mombasa), A1: Pm associated with shrub species Ll ( $L$. leucocephala), Cc (C. cujete), A2: Pm associated with shrub species L and Cc plus tree species Gu (G. ulmifolia), Cg (C. grandis), As (A. saman). Gray bars represent the means of the species evaluated within each arrangement, equal letters not significant, black line experimental error (EE).

La DLR mostró mayores valores en la capa de suelo 0-10 cm, con $201.75 \pm 133.68 \mathrm{~cm} \mathrm{~cm}^{-2}$, y representó el $45 \%$ de la longitud total. Este valor es significativamente diferente en relación con las otras dos capas de suelo $(10-20$ y $20-30 \mathrm{~cm})$, con comportamiento similar $(154.7 \pm 88.4$ y $136.7 \pm 86.36 \mathrm{~cm} \mathrm{~cm}^{-3}$, respectivamente) (Figura 5).

\begin{tabular}{|c|c|c|c|c|c|}
\hline 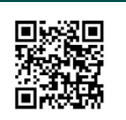 & (c) (i) () () & $\overbrace{\text { AMBINTALES }}$ & $\frac{O \%}{2 \%}$ & 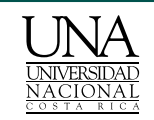 & 60 \\
\hline
\end{tabular}




\section{Revista de CIENCIAS AMBIENTALES Tropical Journal of Environmental Sciences}

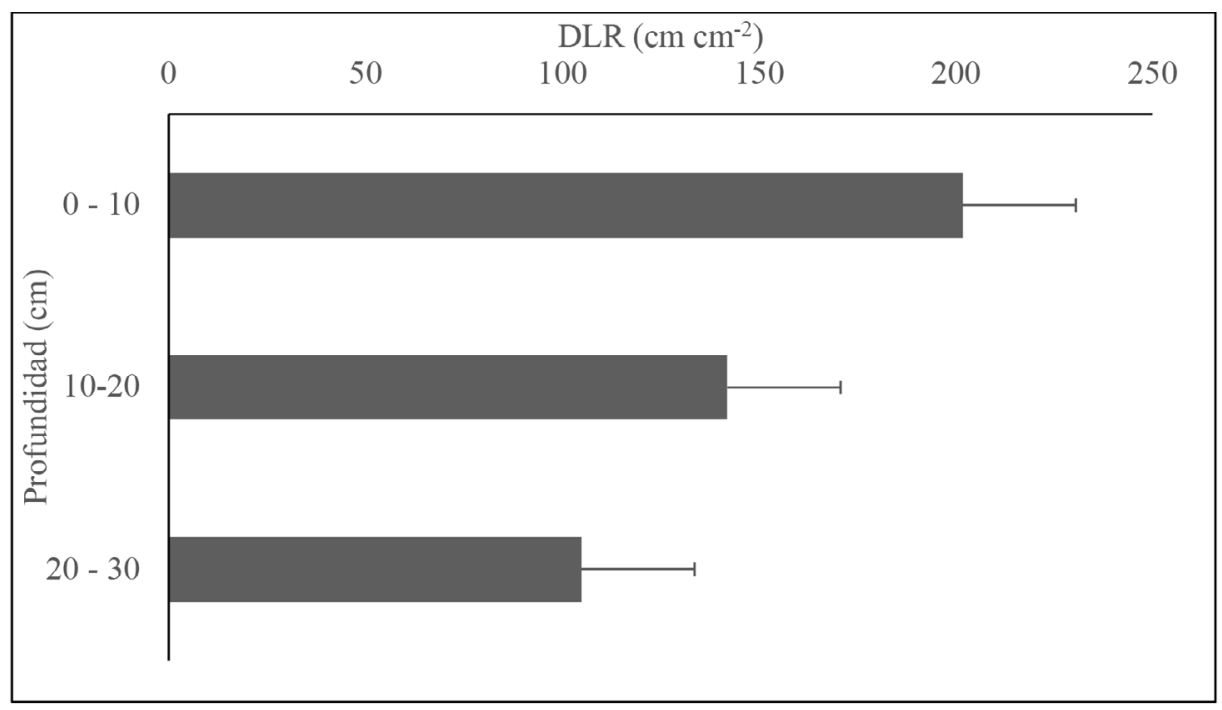

Figura 5. Comportamiento DLR vs profundidad de suelo. A0: Pm (gramínea M. maximus cv Mombasa); A1: Pm asociado con arbustos forrajeros $\mathrm{Ll}$ (L. leucocephala), Cc (C. cujete), A2: Pm asociado con arbustos forrajeros $\mathrm{Ll}$, Cc y árboles forrajeros $\mathrm{Gu}$ (G. ulmifolia), $\mathrm{Cg}$ (C. grandis), As (A. saman). Las barras grises representan las medias de las especies evaluadas dentro de cada arreglo, letras iguales no significancia, línea negra error experimental (EE)

Figure 5. DLR behavior vs. soil depth. A0: Pm (Grass M. maximus cv Mombasa), A1: Pm associated with shrub species Ll ( L. leucocephala), Cc (C. cujete), A2: Pm associated with shrub species $\mathrm{Ll}$ and Cc plus tree species Gu (G. ulmifolia), $\mathrm{Cg}$ (C. grandis), As (A. saman). Gray bars represent the means of the species evaluated within each arrangement, equal letters not significant, black line experimental error (EE)

\subsection{Variables fisicoquímicas de suelo y su correlación con el carbono acumulado en la biomasa de raíces (CBRF) y densidad de longitud de raíces finas (DLR)}

Los resultados obtenidos en las variables edáficas indican que la densidad aparente $(\mathrm{Da})$ y porosidad total $(\mathrm{Pt})$ no presentaron diferencias significativas $(\mathrm{p}>0.05)$ entre los arreglos. Los valores de Da mostraron que el arreglo sin árboles (A0) presenta valores elevados $(1.24 \pm 0.24 \mathrm{~g}$ $\mathrm{cm}^{-3}$ ), con respecto a los registros en los arreglos que incluyen gramíneas y especies arbustivas y arbóreas $\left(1.20 \pm 0.07\right.$ y $\left.1.23 \pm 0.08 \mathrm{~g} \mathrm{~cm}^{-3}\right)$. La variable Pt reportó el menor valor dentro del A0, con disminución del $3 \%$ respecto al arreglo A2 (54.05 $\pm 3.40 \%)$.

Los resultados de $\mathrm{Da}$ y $\mathrm{Pt}$, en profundidad, mostraron significancia $(\mathrm{p}<0.05)$, independiente del arreglo. En la profundidad de $0-10 \mathrm{~cm}$, se evidenció el menor y mayor valor de $\mathrm{Da}$ y $\mathrm{Pt}$ $\left(1.19 \pm 0.08 \mathrm{~g} \mathrm{~cm}^{-3}\right.$ y $54.81 \pm 3.74 \%$, respectivamente). Seguidamente de $10-20 \mathrm{~cm}$, la Da fue mayor (1.26 $\pm 0.19 \mathrm{~g} \mathrm{~cm}^{-3}$ y $51.7 \pm 4.64 \%$, respectivamente), lo cual indica relación inversa entre estas variables (Cuadro 2).

\begin{tabular}{|c|c|c|}
\hline 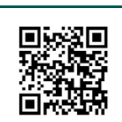 & (c) (i) () (2) & 61 \\
\hline
\end{tabular}




\section{Revista de CIENCIAS AMBIENTALES Vo Tropical Journal of Environmental Sciences}

Revista de Ciencias Ambientales (Trop J Environ Sci) e-ISSN: 2215-3896

(Enero-Junio, 2021). Vol 55(1): 52-69 DOI: https://doi.org/10.15359/rca.55-1.3

Open Access: www.revistas.una.ac.cr/ambientales e-mail: revista.ambientales@una.ac.cr Contreras-Santos J . L., Martínez-Atencia J., Falla-Guzman C. K.

Cuadro 2. Propiedades físicas del suelo dentro de los arreglos y profundidades evaluados en el valle medio del río Sinú, 2012.

Table 2. Physical properties of the soil within the livestock arrangements and depths evaluated in the middle valley of the Sinú river, 2012.

\begin{tabular}{lll}
\hline Arreglo & $\mathrm{Da}\left(\mathrm{g} \mathrm{cm}^{-3}\right)$ & Pt (\%) \\
\hline A0 & $1.24 \pm 0.24$ & $52.73 \pm 3.54$ \\
A1 & $1.20 \pm 0.07$ & $52.92 \pm 4.99$ \\
A2 & $1.23 \pm 0.08$ & $54.05 \pm 3.40$ \\
Prof. & & \\
(cm) & & \\
$0-10$ & $1.19 \pm 0.08 \mathrm{a}^{\star *}$ & $54.81 \pm 3.74 \mathrm{a}^{*}$ \\
$10-20$ & $1.26 \pm 0.19 \mathrm{~b} *$ & $51.7 \pm 4.64 \mathrm{~b}^{\star}$ \\
$\mathrm{R}^{2}$ & 0.39 & 0.23 \\
$\mathrm{CV}$ & 5.1 & 7.2 \\
\hline
\end{tabular}

Letras iguales no existe diferencias significativas; ${ }^{*} \mathrm{p}<0.05 ;{ }^{* *} \mathrm{p}<0.001 ; \mathrm{CV}$ : Coeficiente de variación.

El Cuadro 3 muestra el comportamiento de las propiedades químicas de suelo. El pH, azufre $(\mathrm{S})$, Calcio $(\mathrm{Ca})$, potasio $(\mathrm{K})$, Sodio $(\mathrm{Na})$ y la capacidad de intercambio catiónico $(\mathrm{CIC})$ presentaron diferencias estadísticas significativas $(\mathrm{p}<0.05)$ entre los arreglos evaluados.

$\mathrm{El} \mathrm{pH}$ presentó disminución de iones hidrógeno $\left(\mathrm{H}^{+}\right)$cuando aumentó la complejidad del arreglo, pasando de condición ácida (5.44 \pm 0.54$)$ dentro de A0 a moderadamente ácida (6.03 $\pm 0.22)$ en $\mathrm{A} 2$, las bases intercambiables (Ca y K) aumentaron de óptimo (13.86 $\pm 2.77 ; 0.83 \pm$ $\left.0.24 \mathrm{cmol}_{(+)} \mathrm{kg}^{-1}\right)$ a alto $\left(16.41 \pm 1.79 ; 1.25 \pm 0.53 \mathrm{cmol}_{(+)} \mathrm{kg}^{-1}\right)$ y fueron mayores dentro de los arreglos silvopastoriles (A1 y A2). El S y Na presentaron comportamiento contrario al Ca y K, disminuyendo cuando aumentó la complejidad de los arreglos, con el mayor valor en el arreglo A0 (145.81 $\left.\pm 187.63 \mathrm{mg} \mathrm{kg}^{-1} ; 0.38 \pm 0.03 \mathrm{cmol}_{(+)} \mathrm{kg}^{-1}\right)$, y el menor valor en el arreglo A2 (83 y $47 \%$ respectivamente). Dentro de los arreglos evaluados se evidenciaron correlaciones significativas $(\mathrm{p}<0.001)$ entre las variables químicas de suelo encontrando que el $\mathrm{pH}$ se correlaciona negativamente con los contenidos azufre $(\mathrm{r}=-0.81)$ y Sodio $(\mathrm{r}=-0.92)$; y positivamente con el $\mathrm{Ca}(\mathrm{r}=0.83)$ y $\mathrm{K}(\mathrm{r}=0.71)$. La materia orgánica $\mathrm{MO}$ se correlacionó con los contenidos de $\mathrm{Mg}$ $(\mathrm{r}=0.75)$ y CIC $(\mathrm{r}=0.72)$. El S evidencia correlación negativa inversa con el Ca $(\mathrm{r}=0.73)$ y positiva con el $\mathrm{Na}(\mathrm{r}=0.91)$. El P presenta correlación positiva con el $\mathrm{K}(\mathrm{r}=0.82)$.

\begin{tabular}{|c|c|c|}
\hline 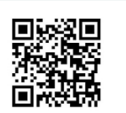 & (c) (1) $(9)$ & 62 \\
\hline
\end{tabular}




\section{Revista de CIENCIAS AMBIENTALES Tropical Journal of Environmental Sciences}

Revista de Ciencias Ambientales (Trop J Environ Sci) e-ISSN: 2215-3896 (Enero-Junio, 2021) . Vol 55(1): 52-69 DOI: https://doi.org/10.15359/rca.55-1.3 Open Access: www.revistas.una.ac.cr/ambientales e-mail: revista.ambientales@una.ac.cr Contreras-Santos J . L., Martínez-Atencia J., Falla-Guzman C. K.

Cuadro 3. Propiedades químicas de suelos en arreglos ganaderos en el valle medio del río Sinú, 2012.

Table 3. Chemical properties of the soil within the livestock arrangements in the middle valley of the Sinú River, 2012.

\begin{tabular}{|c|c|c|c|c|c|c|c|}
\hline \multirow{2}{*}{$\begin{array}{l}\text { Variables } \\
\text { Químicas de } \\
\text { Suelo }\end{array}$} & \multicolumn{3}{|l|}{ Arreglos } & \multirow[b]{2}{*}{$\mathbf{R}^{2}$} & \multirow[b]{2}{*}{$\mathrm{CV}$} & \multirow{2}{*}{$\begin{array}{l}\text { Dev } \\
\text { std }\end{array}$} & \multirow[b]{2}{*}{ Sig } \\
\hline & A0 & A1 & A2 & & & & \\
\hline $\mathrm{pH}$ & $5.44 \pm 0.54 b$ & $5.79 \pm 0.45 \mathrm{ab}$ & $6.01 \pm 0.22 \mathrm{a}$ & 0.31 & 7.45 & 0.48 & $* *$ \\
\hline MO (\%) & $3.87 \pm 0.71 \mathrm{a}$ & $4.15 \pm 0.91 \mathrm{a}$ & $4.17 \pm 0.81 \mathrm{a}$ & 0.13 & 19.95 & 0.81 & $\mathrm{~ns}$ \\
\hline$S\left(\mathrm{mg} \mathrm{kg}^{-1}\right)$ & $\begin{array}{l}145.81 \pm \\
187.63 \mathrm{a}\end{array}$ & $\begin{array}{l}34.38 \pm 20.86 \\
b\end{array}$ & $\begin{array}{l}24.41 \pm 12.37 \\
\mathrm{~b}\end{array}$ & 0.25 & 164.69 & 120.36 & $* *$ \\
\hline $\mathrm{P}\left(\mathrm{mg} \mathrm{kg}^{-1}\right)$ & $\begin{array}{l}18.03 \pm 8.15 \\
\mathrm{a}\end{array}$ & $19.3 \pm 9.10 \mathrm{a}$ & $23.9 \pm 11.14 \mathrm{a}$ & 0.11 & 48.01 & 9.67 & $\mathrm{~ns}$ \\
\hline $\mathrm{Ca}\left(\mathrm{cmol}_{(+)} \mathrm{kg}^{-1}\right)$ & $\begin{array}{l}13.86 \pm 2.77 \\
b\end{array}$ & $13.97 \pm 2.36 b$ & $16.41 \pm 1.79 \mathrm{a}$ & 0.22 & 16.6 & 2.58 & * \\
\hline $\mathrm{Mg}\left(\mathrm{cmol}_{(+)} \mathrm{kg}^{-1}\right)$ & $\begin{array}{l}14.63 \pm 2.30 \\
\mathrm{a}\end{array}$ & $15.31 \pm 2.36 \mathrm{a}$ & $17.05 \pm 4.40 \mathrm{a}$ & 0.2 & 29.11 & 4.40 & $\mathrm{~ns}$ \\
\hline $\mathrm{K}\left(\mathrm{cmol}_{(+)} \mathrm{kg}^{-1}\right)$ & $0.83 \pm 0.33 a$ & $1.19 \pm 0.49 \mathrm{~b}$ & $1.25 \pm 0.53 \mathrm{~b}$ & 0.2 & 43.38 & 0.49 & * \\
\hline $\mathrm{Na}\left(\mathrm{cmol}_{(+)} \mathrm{kg}^{-1}\right)$ & $0.38 \pm 0.24 \mathrm{a}$ & $0.28 \pm 0.09 \mathrm{ab}$ & $0.20 \pm 0.03 b$ & 0.21 & 54.45 & 0.16 & * \\
\hline $\mathrm{CIC}\left(\mathrm{cmol}_{(+)} \mathrm{kg}^{-1}\right)$ & $30.80 \pm 3.20 \mathrm{~b}$ & $31.54 \pm 3.92 \mathrm{ab}$ & $34.91 \pm 4.57 \mathrm{a}$ & 0.21 & 13.45 & 4.57 & * \\
\hline
\end{tabular}

MO: materia orgánica; S: azufre; P: fósforo; Ca: calcio; Mg: magnesio; K: potasio; Na: sodio; CIC: capacidad de intercambio catiónico efectivo; ns: no significativo; ${ }^{*} \mathrm{p}<0.05$ : significativo; ${ }^{* *} \mathrm{p}<0.001$ : altamente significativo.

Se encontró correlación significativa $(\mathrm{p}<0.05)$ entre la Da respecto a la CBRF $(\mathrm{r}=-0.37)$ y DLR ( $r=-0.32)$, lo que indica que cuando aumenta la $\mathrm{Da}$, disminuyen estos valores. Se evidenció correlación significativa $(\mathrm{p}<0.05)$ entre los contenidos de materia orgánica $(\mathrm{MO})$ y potasio $(\mathrm{K})$ del suelo respeto a la CBRF $(\mathrm{r}=0.45,0.73$, respectivamente) y DLR $(\mathrm{r}=0.5,0.69$, respectivamente).

\begin{tabular}{|c|c|c|}
\hline 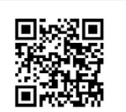 & (c) (1) (9) & 63 \\
\hline
\end{tabular}




\section{Revista de CIENCIAS AMBIENTALES Tropical Journal of Environmental Sciences}

Revista de Ciencias Ambientales (Trop J Environ Sci) e-ISSN: 2215-3896 (Enero-Junio, 2021) . Vol 55(1): 52-69 DOI: https://doi.org/10.15359/rca.55-1.3

Open Access: www.revistas.una.ac.cr/ambientales e-mail: revista.ambientales@una.ac.cr Contreras-Santos J . L., Martínez-Atencia J., Falla-Guzman C. K.

\section{Discusión}

Los mayores contenidos de carbono orgánico en el suelo se dan por acumulación de material muerto (hojas, raíces, ramas y frutos) y rizodeposición (Alonso, 2011; Céspedes Florez et al., 2012).

La biomasa de raíces finas (BRF) de las especies arbustivas y arbóreas se mantiene constante dentro de los arreglos silvopastoriles (SP). Contrario en la gramínea con reducción de BRF del 25 al $60 \%$ respecto al arreglo A0 (Figura 2). Esto puede estar asociado a la capacidad de adquisición de agua y nutrientes de estas especies, coligado a la estructura radicular pivotante, capaz de adquirir nutrimentos de estratos profundos. La gramínea presenta sistema radicular fasciculado capaz de desarrollarse en los primeros $30 \mathrm{~cm}$ de suelo, impidiéndole competir de manera eficiente por nutrimentos y agua. Resultados similares reporta Casanova et al. (2010), al encontrar que el aumento de especies dentro de los sistemas disminuye la biomasa radical de la gramínea. Esto se relaciona con la disponibilidad de nutrientes, luz, agua y tasa de consumo de las especies presentes (Davis et al., 1999).

Diferentes estudios han encontrado que la biomasa radicular de las especies arbustivas y arbóreas aporta más del $40 \%$ de la biomasa total de los arreglos silvopastoriles (Casanova et al., 2010; López et al., 2018). El sistema radicular de las plantas es considerado un importante reservorio de carbono, debido a su rápida reconversión (senescencia); el asocio de gramíneas más especies arbóreas mejoran la acumulación de carbono en el suelo en estratos superficiales y subsuperficiales, producto de la bioturbación (Casals et al. 2014); valores bajos de BRF son indicadores de suelos degradados y bajo contenido de C (Casanova et al., 2010; Nair et al., 2009; Rojas et al., 2009).

El stock de carbono acumulado en la BRF dentro de los arreglos disminuyó conforme se profundiza en el perfil de suelo (Figura 3), y evidenció la mayor acumulación en los primero 10 $\mathrm{cm}$ de suelo. Estos valores están asociados al aumento de la densidad aparente del suelo en estratos subsuperficiales. Resultados similares reporta Céspedes Flores et al. (2012), en diferentes sistemas de pastoreo, donde encontró que el mayor stock de carbono acumulado en la biomasa de raíces se presenta en los primeros $20 \mathrm{~cm}$ de suelo y disminuye en profundidad. Valores altos de CBRF están asociados a buenas condiciones fisicoquímicas de suelo, relacionadas con buena aireación (ej. alta porosidad, baja densidad aparente), disponibilidad de nutrientes y agua (Barreto - Sánchez et al. 2005; Casals et al. 2014; Contreras-Santos et al., 2019; Jiménez et al. 2004; Nair et al., 2009).

La densidad de longitud de raíces finas (DLR) reportó valores altos dentro de las especies arbustivas y arbóreas evaluadas (Figura 4), esto se relaciona con la capacidad de estas especies para explorar el suelo en búsqueda de nutrientes y agua (Bowen 1985). Los arreglos silvopastoriles A1 y A2 presentaron mayor DLR de raíces finas que el arreglo de solo gramínea sin árboles (A0). En investigación realizada por Kumar et al., (2010), encontraron que la mayor DLR se presentó en los suelos que asociaban gramíneas más árboles. Estos resultados evidencian la capacidad que tienen los arreglos agroforestales y silvopastoriles en la recuperación de suelo

\begin{tabular}{|c|c|c|}
\hline 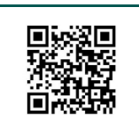 & (c) (1) () (2) (2) & 64 \\
\hline
\end{tabular}




\section{Revista de CIENCIAS AMBIENTALES Tropical Journal of Environmental Sciences}

Revista de Ciencias Ambientales (Trop J Environ Sci) e-ISSN: 2215-3896 (Enero-Junio, 2021). Vol 55(1): 52-69 DOI: https://doi.org/10.15359/rca.55-1.3

Open Access: www.revistas.una.ac.cr/ambientales e-mail: revista.ambientales@una.ac.cr Contreras-Santos J . L., Martínez-Atencia J., Falla-Guzman C. K.

(Hertel et al 2003). La profundidad del perfil de suelo influyó en la DLR en los arreglos (Figura 5), al registrar los valores más elevados en el estrato superficial; similares resultados dan Schroth (1999) y Kumar et al., (2010), quienes reportaron que los valores de DLR se reducen al aumentar la profundidad de suelo. En la investigación realizada por Gaitán et al. (2005) observaron que la mayor concentración de las raíces finas de Eucalyptus globulos se presentó en los primeros 20 $\mathrm{cm}$, lo que está asociado a mayor concentración de nutrientes y agua. Otros estudios, realizados por Kumar et al., (2010), reportan que valores altos de densidad aparente en el suelo, disminuyen la BRF y DLR. Investigaciones realizadas en plantaciones de Eucalytus globulus muestran una relación estrecha entre la distribución de raíces y las propiedades físicas de suelo, y que el aumento de los parámetros físicos de suelo disminuye la producción de BRF y DLR (Gaitan et al., 2005).

Los sistemas que asocian especies arbóreas leñosas y pasturas son capaces de aportar altos contenidos de materia orgánica al suelo, y contribuyen a mejorar las propiedades físicas (Contreras-Santos et al., 2019; Leyva et al., 2018). Igualmente reportan que el uso de prácticas sostenibles en suelos ganaderos mejora las condiciones de aireación, disminuye la compactación en estratos superficiales y genera condiciones adecuadas para el desarrollo de las raíces.

Los resultados químicos de suelo (Cuadro 3) obtenidos muestran que los arreglos que incluyen especies arbustivas y arbóreas presentaron menor concentración de hidrogeniones $(\mathrm{H}+)$, igualmente aumentaron los valores de $\mathrm{MO}, \mathrm{P}$ disponible, $\mathrm{Ca}^{2+} \mathrm{y} \mathrm{K}^{+}$extraíbles, resultados similares a los reportados por Casals et al., (2014), en arreglos que asocian pasturas más arboles dispersos. El aumento de las bases intercambiables $\mathrm{Ca}^{2+}$ y $\mathrm{K}^{+}$explican la reducción de concentración de $\mathrm{H}^{+}$en suelo (Casals et al. 2014). Los altos valores de BRF y DLR dentro de los arreglos que asocian especies arbustivas y arbóreas, pueden ser relacionados con alta concentración de nutrimentos disponibles dentro de los arreglos silvopastoriles, generado por la acumulación de material orgánico, a causa de la caída de hojarasca, ramas, troncos y residuos orgánicos producto de las excretas de los animales.

\section{Conclusiones}

El asocio de gramíneas con especies arbustivas y arbóreas en un arreglo silvopastoril aumenta la cantidad de raíces finas, el stock de carbono (C) acumulado y mejora las características fisicoquímicas del suelo. El mayor stock de $\mathrm{C}$ acumulado en la biomasa de raíces se evidenció en los primeros $10 \mathrm{~cm}$ de profundidad en todos los arreglos. Se destacan las especies arbustivas (L. leucocephala y C. cujete) por exhibir los mayores valores de stock de carbono acumulado en la biomasa de raíces finas y densidad de raíces finas.

\section{6. Ética y conflicto de intereses}

Las personas autoras declaran que han cumplido totalmente con todos los requisitos éticos y legales pertinentes, tanto durante el estudio como en la producción del manuscrito; que no hay

\begin{tabular}{|c|c|c|c|c|c|}
\hline 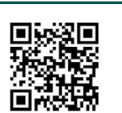 & (c) (1) (1) (2) & 8 & $\frac{2 \%}{20}$ & 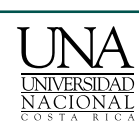 & 65 \\
\hline
\end{tabular}




\section{Revista de CIENCIAS AMBIENTALES Tropical Journal of Environmental Sciences}

Revista de Ciencias Ambientales (Trop J Environ Sci) e-ISSN: 2215-3896 (Enero-Junio, 2021) . Vol 55(1): 52-69 DOI: https://doi.org/10.15359/rca.55-1.3

Open Access: www.revistas.una.ac.cr/ambientales e-mail: revista.ambientales@una.ac.cr Contreras-Santos J . L., Martínez-Atencia J., Falla-Guzman C. K.

conflictos de intereses de ningún tipo; que todas las fuentes financieras se mencionan completa y claramente en la sección de agradecimientos; y que están totalmente de acuerdo con la versión final editada del artículo.

\section{Agradecimientos}

Los autores agradecen a la Corporación Colombiana de Investigación Agropecuaria (AGROSAVIA) y Ministerio de Agricultura y Desarrollo rural (MADR), por el financiamiento de esta investigación, desarrollada dentro del proyecto "Valoración integral de los beneficios de sistemas silvopastoriles de estratos múltiples sobre la sostenibilidad del suelo y la productividad animal en el sistema doble propósito de la región Caribe”. Finalmente, agradecemos a la Revista y las personas especialistas que dictaminaron la versión final del documento, por sus valiosas contribuciones.

\section{Referencias}

Alonso, J. (2011). Los sistemas silvopastoriles y su contribución al medio ambiente. Revista Cubana de Ciencia Agrícola, 45(2), 107-115.

Baker, T. T., Conner, W. H., Lockaby, B. G., Stanturf, J. A., y Burke, M. K. (2002). Fine root productivity and dynamics on a forested floodplain in south Carolina. Soil Sci. Soc. Am. J., 66(2), 545-556. https://doi.org/10.2136/sssaj2002.0671

Barreto - Sánchez, L. H., León, P. J. (2005). Masa total y contenido de nutrientes en raíces finas de ecosistemas forestales (pinuspatulaschltdl y chamcupressuslusitanicamill y quercushumboldtiibonpl.) De piedras blancas, antioquia-colombia. Revista Facultad Nacional de Agronomía Medellín, 58(2), 2907-2929.

Bowen, G.D. (1985) Roots as a component of tree productivity. In M. G. R. Cannell, J. E. Jackson (Eds), Attributes of trees as crop plants (pp 303-315). Institute of Terrestrial Ecology Natural Environment Research Council.

Burke, M. K., y Raynal, D. J. (1994). Fine root growth phenology, production, and turnover in a northern hardwood forest ecosystem. Plant and Soil, 162(1), 135-146. https://doi. org/10.1007/BF01416099

Casals, P., Romero, J., Rusch, G. M., y Ibrahim, M. (2014). Soil organic C and nutrient contents under trees with different functional characteristics in seasonally dry tropical silvopastures. Plant and Soil, 374(1-2), 643-6599. https://doi.org/10.1007/s11104-013-1884-9

Casanova - Lugo, F., Maldonado, J. C., Aldana, J. P., Sánchez, F. S., y Caamal, J. C. (2010). Acumulación de carbono en la biomasa de Leucaena leucocephala y Guazuma ulmifolia asociadas y en monocultivo. La Revista Forestal Venezolana, 54(1), 45-51.

\begin{tabular}{|c|c|c|c|c|c|}
\hline 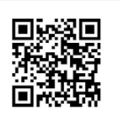 & (c) (1) (9) & $\underset{\text { AMBIENTAIES }}{\Leftrightarrow}$ & $\frac{10 \%}{\frac{9}{2}}$ & 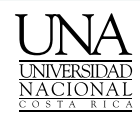 & 66 \\
\hline
\end{tabular}




\section{Revista de CIENCIAS AMBIENTALES Tropical Journal of Environmental Sciences} Revista de Ciencias Ambientales (Trop J Environ Sci)
e-ISSN: 2215-3896
(Enero-Junio, 2021) .Vol 55(1): 52-69
DOI: https://doi.org/10.15359/rca.55-1.3
Open Access: www.revistas.una.ac.cr/ambientales
e-mail: revista.ambientales@una.ac.cr Contreras-Santos J . L., Martínez-Atencia J., Falla-Guzman C. K.

Cajas-Giron, Y. S., Jones, M., y Sinclair, F. L. (2002). Combining tree diversity and cattle productivity on seasonally dry pastures in Columbia. International conference on responding to the increasing global demand for animal products. British Society of Animal Science.

Céspedes Flores, F. E., Fernández, J. A., Gobbi, J. A., y Bernardis, A. C. (2012). Reservorio de carbono en suelo y raíces de un pastizal y una pradera bajo pastoreo. Revista fitotecnia mexicana, 35(1), 79-86.

Contreras-Santos, J. L., J., Martínez Atencia, J., Cadena Torres, y C. K., Fallas Guzmán. (2019). Evaluación del carbono acumulado en suelo en sistemas silvopastoriles del Caribe Colombiano. Agronomía Costarricense, 44(1), 29-41. https://doi.org/10.15517/rac.v44i1.39999

Davis, M. A., Wrage, K. J., Reich, P. B., Tjoelker, M. G., Schaeffer, T., y Muermann, C. (1999). Survival, growth, and photosynthesis of tree seedlings competing with herbaceous vegetation along a water-light-nitrogen gradient. Plant Ecology, 145(2), 341-350. https://doi. org/10.1023/A:1009802211896

Organización de las Naciones Unidas para la Alimentación y la Agricultura (FAO) y Grupo Técnico intergubernamental de Suelos (GTIS). (2015). Estado Mundial del Recurso Suelo (EMRS) - Resumen Técnico. Grupo Técnico Intergubernamental del Suelo y Organización de las Naciones Unidas para la Alimentación y la Agricultura. Organización de las Naciones Unidas para la Alimentación y la Agricultura y Grupo Técnico. http://www.fao.org/3/ai5126s.pdf

Gaitán, J. J., Penón, E. A., y Costa, M. C. (2005). Distribución de raíces finas de Eucalyptus globulus ssp. maidenii y su relación con algunas propiedades del suelo. Ciência Florestal, 15(1), 33-41. http://dx.doi.org/10.5902/198050981822

Hertel, D., Leuschner, C., y Holscher, D. (2003). Size and Structure of Fine Root Systems in Old-growth and Secondary Tropical Montane Forests (Costa Rica). Biotropica, 35(2), 143153. https://doi.org/10.1111/j.1744-7429.2003.tb00274.x

Holdridge, L. (2000). Ecología basada en zonas de vida. Instituto Interamericano de Cooperación para la Agricultura, IICA.

International Organization for Standardization (ISO). (2016). Animal feeding stuffs: Determination of moisture and other volatile matter content (ISO 6496, $2^{\mathrm{da}} \mathrm{ed} ., 1-13 \mathrm{pp}$.). ISO.

Instituto Geográfico Agustín Codazzi IGAC, (2006). Métodos analíticos del laboratorio de suelo (Sexta edición). Imprenta Nacional.

Jiménez-Rodríguez, C., y Arias-Aguilar, D. (2004). Distribución de la biomasa y densidad de raíces finas en una gradiente sucesional de bosques en la Zona Norte de Costa Rica. Revista

\begin{tabular}{|c|c|c|c|c|c|}
\hline 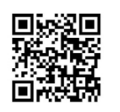 & (c) $\underset{\mathrm{BY}}{(1)}(0)$ & $\underset{\text { AMBENETILES }}{3}$ & $\begin{array}{l}\frac{O O}{2} \\
\frac{2}{2} \\
\text { euna }\end{array}$ & 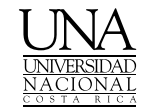 & 67 \\
\hline
\end{tabular}




\section{Revista de CIENCIAS AMBIENTALES Tropical Journal of Environmental Sciences}

Revista de Ciencias Ambientales (Trop J Environ Sci) e-ISSN: 2215-3896 (Enero-Junio, 2021). Vol 55(1): 52-69 DOI: https://doi.org/10.15359/rca.55-1.3

Open Access: www.revistas.una.ac.cr/ambientales e-mail: revista.ambientales@una.ac.cr Contreras-Santos J . L., Martínez-Atencia J., Falla-Guzman C. K.

Forestal Mesoamericana Kurú, 1(2), 44-63. https://revistas.tec.ac.cr/index.php/kuru/article/ view/572

Jose, S., y Dollinger, J. (2019). Silvopasture: a sustainable livestock production system. Agroforestry Systems, 93(1), 1-9. https://doi.org/10.1007/s10457-019-00366-8

Kumar, S., Udawatta, R. P., y Anderson, S. H. (2010). Root length density and carbon content of agroforestry and grass buffers under grazed pasture systems in a Hapludalf. Agroforestry Systems, 80(1), 85-96. https://doi.org/10.1007/s10457-010-9312-0

Leyva, S., Baldoquin, A. Reyes, M. (2018). Propiedades de los suelos en diferentes usos agropecuarios, Las Tunas, Cuba. Rev. Cienc. Agr., 35(1), 36-47. http://dx.doi.org/10.22267/ rcia. 183501.81

López-Santiago, J. G., Casanova-Lugo, F., Villanueva-López, G., Díaz-Echeverría, V. F., Solorio-Sánchez, F. J., Martínez-Zurimendi, P., ... Chay-Canul, A. J. (2018). Carbon storage in a silvopastoral system compared to that in a deciduous dry forest in Michoacán, Mexico. Agroforestry Systems, 93(1), 199-211. https://doi.org/10.1007/s10457-018-0259-x

Nair, P.K.R, Kumar, B.M., y Nair, V.D. (2009). Agroforestry as a strategy for carbon sequestration. Journal of Plant Nutrition and Soil Science, 172(1), 10-23. https://doi.org/10.1002/ jpln.200800030

Pérez-Ramírez, S., Ramírez, M. I., Jaramillo-López, P. F., y Bautista, F. (2013). Contenido de carbono orgánico en el suelo bajo diferentes condiciones forestales: Reserva de la biosfera mariposa monarca, México. Revista Chapingo Serie Ciencias Forestales y Del Ambiente, 19(1), 157-173. http://dx.doi.org/10.5154/r.rchscfa.2012.06.042.

Quiceno-Urbina, N.-J., Tangarife-Marín, G.-M., y Álvarez-León, R. (2016). Estimación del contenido de biomasa, fijación de carbono y servicios ambientales, en un área de bosque primario en el resguardo indígena Piapoco Chigüiro-chátare de Barrancominas, departamento del Guainía (Colombia). Luna Azul, 43, 171-202. http://dx.doi.org/10.17151/luaz.2016.43.9.

Rojas M., J., Ibrahim, M., y Andrade, H. (2009). Secuestro de carbono y uso de agua en sistemas silvopastoriles con especies maderables nativas en el trópico seco de Costa Rica. Ciencia y Tecnología Agropecuaria, 10(2), 214-223. https://doi.org/10.21930/rcta.vol10_num2_art:144

Schroth, G. (1999). A review of belowground interactions in agroforestry, focussing on mechanisms and management options. Forestry Sciences, 43, 5-34. https://doi. org/10.1023/A:1026443018920

Schroth, G., y Zech, W. (1995). Root length dynamics in agroforestry with Gliricidia sepium as compared to sole cropping in the semi-deciduous rainforest zone of West Africa. Plant and Soil, 170(2), 297-306. https://doi.org/10.1007/BF00010482
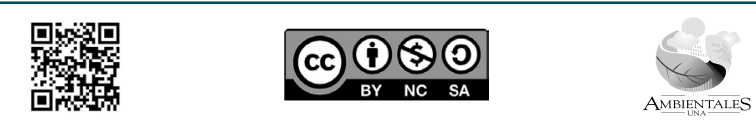


\section{Revista de CIENCIAS AMBIENTALES

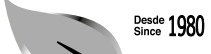

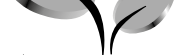 \\ Tropical Journal of Environmental Sciences}

Revista de Ciencias Ambientales (Trop J Environ Sci) e-ISSN: 2215-3896

(Enero-Junio, 2021) . Vol 55(1): 52-69 DOI: https://doi.org/10.15359/rca.55-1.3

Open Access: www.revistas.una.ac.cr/ambientales e-mail: revista.ambientales@una.ac.cr Contreras-Santos J . L., Martínez-Atencia J., Falla-Guzman C. K.

Silver, W. L., y Miya, R. K. (2001). Global patterns in root decomposition: comparisons of climate and litter quality effects. Oecologia, 129(3), 407-419. https://doi.org/10.1007/s004420100740

Sotelo, M., Suárez-Salazar, J. C., Álvarez, F., Castro Núñez, A., Calderón-Soto, V. H., Arango, J. (2017). Sistemas sostenibles de producción ganadera en el contexto amazónico - Sistemas silvopastoriles: ¿una opción viable? Publicación CIAT No. 448. Centro Internacional de Agricultura Tropical (CIAT. https://hdl.handle.net/10568/89088 